\title{
Corrigendum: Table of contents
}

Nat. Genet. 37 (7), i (2005).

The cover art for the July issue was "miRNA” by Scott Bodel, provided by Rosetta Genomics.

\section{Corrigendum: An adaptive radiation model for the origin of new gene}

일

M P Francino

Nat. Genet. 37, 573-577 (2005).

The author also thanks the University of California Lawrence Berkeley National Laboratory for support. 